\title{
Min-Max Quickest Path Problems
}

\author{
Stefan Ruzika* and Markus Thiemann* \\ University of Kaiserslautern, Postbox 3049, 67653 Kaiserslautern, Germany. \\ \{ruzika, thiemann\}@mathematik.uni-kl.de
}

November 18, 2010

\begin{abstract}
In a dynamic network, the quickest path problem asks for a path such that a given amount of flow can be sent from source to sink via this path in minimal time. In practical settings, for example in evacuation or transportation planning, the problem parameters might not be known exactly a-priori. It is therefore of interest to consider robust versions of these problems in which travel times and/or capacities of arcs depend on a certain scenario. In this article, min-max versions of robust quickest path problems are investigated and, depending on their complexity status, exact algorithms or fully polynomial-time approximation schemes are proposed.
\end{abstract}

Keywords: quickest path problem, robust network flows, optimization, fptas, polynomial algorithms, multiple objective optimization

\section{Introduction}

The quickest path problem is a generalization of the shortest path problem in dynamic networks in which flow units are assumed to take time to traverse an arc. Given an amount of flow $U$ and two nodes $s$ and $t$, the goal of the quickest path problem is to find an $s$-t-path with minimum transmission time, that is the sum of the travel time from $s$ to $t$ of this path and the number of repetitions to send all $U$ flow units along this path. Applications of this problem can be found in communication networks, transportation networks, and evacuation modeling $[5,7,13]$. The quickest path problem was formally introduced by Chen and Chin [4] who also presented a polynomialtime solution algorithm by reducing it to the shortest path problem in a modified network. Other authors also derived polynomial-time algorithms

\footnotetext{
*Partially supported by BMBF, Project REPKA, FKZ 13N9961 (TU KL).
} 
for this problem $[12,17]$. Numerous variants and extensions of the quickest path problem have been considered, including all pairs quickest path problems $[8,10]$, the $k$-quickest path problem [3], and most reliable quickest path problems $[11,19]$. More information about quickest path problems can be found in the survey of Pascoal et al. [16].

In practice, model parameters or objective function coefficients are often uncertain or imprecise. This uncertainty can be dealt with by considering different scenarios, each of which corresponds to a specified parameter combination. There are two standard techniques to describe these scenarios. In the interval data technique, each parameter can take values between a lower and an upper bound. In the discrete scenario technique, each scenario for the parameters is described explicitly. This article focuses on the latter case. Kouvelis and $\mathrm{Yu}[9]$ propose min-max problems arising in the discrete scenario case, where solutions with the best worst-case performance under all scenarios are to be found. These min-max problems - as well as the related min-max regret problems (cf. [9]) - are considered as robust optimization problems. In min-max regret problems, solutions with the best worst-case performance relative to the optimal solutions are sought.

Min-max and min-max regret versions of some network optimization problems are examined in the articles $[1,9,20]$. In their survey about minmax combinatorial optimization problems [1], Aissi et al. summarize complexity results for robust shortest path, knapsack, and minimum spanning tree problems. Pseudopolynomial algorithms and approximations are proposed in their follow-up work [2]. There, a general approximation scheme for min-max and min-max regret versions of combinatorial minimization problems is presented. For the min-max and min-max regret shortest path problem, pseudopolynomial algorithms (see also [20]) and fully polynomial-time approximation schemes (assuming the number of scenarios to be constant) are deduced. Since the quickest path problem generalizes the shortest path problem, techniques similar to the ones used in [2] will be utilized in the subsequent disquisition of the min-max quickest path problem.

This article addresses min-max versions of the quickest path problem which - to the best of our knowledge - have not been examined yet. The next section formally introduces the quickest path problem and its min$\max$ version. Since the objective function of the min-max quickest path problem depends on two parameters, the three possible combinations of scenario-dependent parameters are analyzed in this article: Section 3 examines robust quickest path problems with scenario-dependent capacities; min-max quickest path problems with scenario-dependent travel times are solved with pseudopolynomial algorithms and approximated polynomially in Section 4; Section 5 treats the case, where capacities and travel times are simultaneously uncertain. The article is concluded in Section 6 with 
some future research ideas, especially on the min-max regret quickest path problem.

\section{$2 \quad$ Preliminaries}

Let $G=(N, A, \tau, u)$ be a dynamic network with node set $N$, arc set $A$, travel times $\tau_{i j} \in \mathbb{Z}_{0}^{+}$, and capacities $u_{i j} \in \mathbb{N}$ for all arcs $(i, j) \in A$. Let $n$ denote the number of nodes and $m$ the number of arcs in $G$, respectively. The node set is assumed to subsume a source $s \in N$ and a sink $t \in N$. Let $\mathcal{P}$ denote the set of all $s$-t-paths in $G$. Travel time and capacity of a path $P \in \mathcal{P}$ are defined as $\tau(P):=\sum_{(i, j) \in P} \tau_{i j}$ and $u(P):=\min _{(i, j) \in P} u_{i j}$, respectively. For a given amount of flow $U \in \mathbb{N}$, the transmission time of an $s$-t-path $P$ is $\sigma(P):=\tau(P)+\left\lceil\frac{U}{u(P)}\right\rceil$. The Quickest Path Problem asks for an $s$-t-path with minimum transmission time, i.e. a solution of the problem $\min _{P \in \mathcal{P}} \sigma(P){ }^{1}$

Lemma 1 (Rosen et al. [17]). The quickest path problem can be solved in $\mathcal{O}\left(m^{2}+m n \log n\right)$.

Let $S=\{1, \ldots, p\}, p \in \mathbb{N}$, be a set of scenarios. For each scenario $r \in S$, travel times $\tau_{i j}^{r} \in \mathbb{Z}_{0}^{+}$and capacities $u_{i j}^{r} \in \mathbb{N}$ are assumed to be given for all $(i, j) \in A$. Analogously to the definition above, the travel time of a path $P \in \mathcal{P}$ in scenario $r \in S$ is defined as $\tau^{r}(P):=\sum_{(i, j) \in P} \tau_{i j}^{r}$, its capacity is $u^{r}(P):=\min _{(i, j) \in P} u_{i j}^{r}$, and its transmission time is $\sigma^{r}(P):=$ $\tau^{r}(P)+\left\lceil\frac{U}{u^{r}(P)}\right\rceil$.

The goal of the min-max quickest path problem is to find an s-t-path having the minimal worst case transmission time among all scenarios, i.e.,

$$
\min _{P \in \mathcal{P}} \max _{r \in S} \sigma^{r}(P)
$$

In this article, three different types of dependency on scenario sets together with the min-max quickest path problem are considered. If the travel time of each arc is fixed for all scenarios, i.e., it is $\tau_{i j}^{r}=\tau_{i j}$ for all $r \in S$ and $(i, j) \in A$, but the capacities are scenario-dependent, the corresponding quickest path problem is referred to as $\mathcal{Q}_{u}$. In the converse case that capacities on every arc $(i, j) \in A$ are fixed to $u_{i j}$ for all scenarios, but the travel times vary, the resulting quickest path problem is denoted by $\mathcal{Q}^{\tau}$. If both, travel times and capacities, are assumed to be scenario-dependent,

\footnotetext{
${ }^{1}$ In fact, $\tau^{r}(P)+\left[\frac{U}{u^{r}(P)}\right]-1$ represents the time needed to send $U$ units of flow along path $P$, starting at time 0 . Since it is common practice in the literature to omit the constant -1 and since this does not alter the optimal solution of the problem, it is also omitted in this article.
} 
the problem is named $\mathcal{Q}_{u}^{\tau}$. Each of the corresponding min-max problems is referred to as MIN-MAX $\mathcal{Q}$ with $\mathcal{Q} \in\left\{\mathcal{Q}_{u}, \mathcal{Q}^{\tau}, \mathcal{Q}_{u}^{\tau}\right\}$, respectively. If ambiguity can be excluded, the solutions of MIN-MAX $\mathcal{Q}$ problems are simply referred to as min-max quickest paths.

Some concepts of approximation algorithms for optimization problems shall be briefly recalled next (cf. [14]). For an instance $\mathcal{I}$ of a minimization problem $\mathcal{R}$, let $\operatorname{opt}(\mathcal{I})$ be the optimal objective function value and $x$ be a feasible solution with objective function value $\operatorname{val}(x)$. Assume that $\operatorname{opt}(\mathcal{I}) \geq$ 0 . For a constant $c \geq 1, x$ is a c-approximation of $\operatorname{opt}(\mathcal{I})$, if $\operatorname{val}(x) \leq$ $c \cdot \operatorname{opt}(\mathcal{I})$. An algorithm is a c-approximation algorithm for $\mathcal{R}$, if for all instances $\mathcal{I}$ of $\mathcal{R}$, it returns a $c$-approximative solution $x$. An optimization problem has a fully polynomial-time approximation scheme (fptas), if for any constant $\varepsilon>0$ it admits a $(1+\varepsilon)$-approximation algorithm with running time polynomial both in the size of the input and in $\frac{1}{\varepsilon}$.

\section{Scenario-Dependent Capacities}

The min-max quickest path problem with scenario-dependent capacities and fixed travel times is considered first and it is shown that it can be solved by a single quickest path computation in a modified dynamic network using aggregated arc capacities.

Consider the problem Min-Max $\mathcal{Q}_{u}$. Define the dynamic network $G^{\text {min }}=$ $\left(N, A, \tau, u^{\min }\right)$ with $u_{i j}^{\min }:=\min _{r \in S} u_{i j}^{r}$. By definition, capacities are positive integers for all scenarios and, thus, $u_{i j}^{\min } \in \mathbb{N}$ for all $(i, j) \in A$. Hence, the sets of feasible $s$ - $t$-paths in $G$ and $G^{\text {min }}$ coincide.

Lemma 2. Let $Q$ be a quickest s-t-path in $G^{\mathrm{min}}$. Then, $Q$ is a solution of $\operatorname{MIN}-\mathrm{MAX} \mathcal{Q}_{u}$. 
Proof.

$$
\begin{aligned}
\tau(Q)+\left\lceil\frac{U}{u^{\min }(Q)}\right] & =\min _{P \in \mathcal{P}}\left(\tau(P)+\left\lceil\frac{U}{u^{\min }(P)}\right\rceil\right) \\
& =\min _{P \in \mathcal{P}}\left(\tau(P)+\left\lceil\frac{U}{\min _{(i, j) \in P} u_{i j}^{\min }}\right\rceil\right) \\
& =\min _{P \in \mathcal{P}}\left(\tau(P)+\left\lceil\frac{U}{\min _{(i, j) \in P} \min _{r \in S} u_{i j}^{r}}\right\rceil\right) \\
& =\min _{P \in \mathcal{P}}\left(\tau(P)+\left\lceil\frac{U}{\min _{r \in S} \min _{(i, j) \in P} u_{i j}^{r}}\right\rceil\right) \\
& =\min _{P \in \mathcal{P}}\left(\tau(P)+\left\lceil\frac{U}{\min _{r \in S} u^{r}(P)}\right\rceil\right) \\
& =\min _{P \in \mathcal{P}}\left(\tau(P)+\left\lceil\max _{r \in S} \frac{U}{u^{r}(P)}\right\rceil\right) \\
& =\min _{P \in \mathcal{P}} \max _{r \in S}\left(\tau(P)+\left\lceil\frac{U}{u^{r}(P)}\right\rceil\right)
\end{aligned}
$$

The last two equations are valid since $u^{r}(P)>0$ for all $r \in S$ and since $U>0$.

Corollary 1. MIN-MAX $\mathcal{Q}_{u}$ can be solved in $\mathcal{O}\left(m^{2}+m n \log n\right)$.

Proof. Follows directly from Lemma 2 and Lemma 1.

\section{Scenario-Dependent Travel Times}

The min-max quickest path problem with scenario-dependent travel times and fixed capacities is considered next. Observe that for $U=1$, the quickest path problem reduces to a shortest path problem. This observation transfers to the min-max version of the quickest path problem for $U=1$. Thus, MIN-MAX $\mathcal{Q}^{\tau}$ is at least as hard to solve as the corresponding shortest path problem.

Definition 1. A layered network is a network $G=(N, A)$ with the following two properties:

- There exists a partition $N=\{s\} \cup N_{1} \cup N_{2} \cup \ldots \cup N_{k} \cup\{t\}$ with $N_{i} \cap N_{j}=\emptyset, i \neq j$. 
- Arcs only exist from $s$ to $N_{1}$, from $N_{k}$ to $t$, and from $N_{i}$ to $N_{i+1}$ for $i=1, \ldots, k-1$.

Let $\Delta:=\max \left\{\left|N_{i}\right|, i=1, \ldots, k\right\}, \Delta$ is called the width of the layered network.

Yu and Yang [20] show that the min-max shortest path problem is NPhard for layered networks of width 2 with 2 scenarios. The following corollary is an immediate consequence of this result.

Corollary 2. MIN-MAX $\mathcal{Q}^{\tau}$ is NP-hard, even in layered networks of width 2 and with 2 scenarios.

Recall that the task in the min-max shortest path problem is to solve $\min _{P \in \mathcal{P}} \max _{r \in S} \tau^{r}(P)$. Let $B$ be an upper bound on the value of the shortest $s$-t-path for any scenario $r \in S$. Aissi et al. [2] state a pseudopolynomial algorithm for the min-max shortest path problem relying on this bound.

Lemma 3. [Aissi et al. [2]] The min-max shortest path problem for $p$ scenarios can be solved in $\mathcal{O}\left(n^{2} B^{p-1}\right)$.

\subsection{Pseudopolynomial Algorithm for MIN-MAX $\mathcal{Q}^{\tau}$}

Assume that there are $l$ different capacity values $u_{1}<u_{2}<\ldots<u_{l}$ on the $\operatorname{arcs}$ of $G, l \leq m$. For $w>0$, define the network $G(w):=(N, A(w), \tau, u)$, with $A(w):=\left\{(i, j) \in A: u_{i j} \geq w\right\}$. By definition, $G(1)=G$ and every $s$-t-path in $G(w)$ carries at least $w$ flow units.

Lemma 4. Let $P^{\star}$ be a min-max quickest s-t-path in $G$. Then, $P^{\star}$ is a min-max shortest s-t-path in $G\left(u\left(P^{\star}\right)\right)$.

Proof. Let $Q$ be an arbitrary s-t-path in $G\left(u\left(P^{\star}\right)\right)$. Then, $u(Q) \geq u\left(P^{\star}\right)$. Since $P^{\star}$ is a min-max quickest $s$-t-path, it is

$$
\begin{aligned}
\max _{r \in S}\left(\tau^{r}\left(P^{\star}\right)+\left\lceil\frac{U}{u\left(P^{\star}\right)}\right\rceil\right) & =\min _{P \in \mathcal{P}} \max _{r \in S}\left(\tau^{r}(P)+\left\lceil\frac{U}{u(P)}\right\rceil\right) \\
& \leq \max _{r \in S}\left(\tau^{r}(Q)+\left\lceil\frac{U}{u(Q)}\right\rceil\right) .
\end{aligned}
$$

Since $u\left(P^{\star}\right) \leq u(Q)$, it holds $\max _{r \in S} \tau^{r}\left(P^{\star}\right) \leq \max _{r \in S} \tau^{r}(Q)$.

Lemma 5. Let $P_{j}$ be a min-max shortest s-t-path in $G\left(u_{j}\right), j=1, \ldots, l$. Let

$$
P_{k} \in \underset{j=1, \ldots, l}{\operatorname{argmin}} \max _{r \in S} \sigma^{r}\left(P_{j}\right) .
$$

Then, $P_{k}$ is a min-max quickest s-t-path in $G$. 
Proof. Assume $P$ is a min-max quickest $s$ - $t$-path in $G$. By definition, $u(P) \in$ $\left\{u_{1}, \ldots, u_{l}\right\}$, so assume $u(P)=u_{j_{0}}$ for some $j_{0} \in\{1, \ldots, l\}$. Due to Lemma 4, $P$ and $P_{j_{0}}$ are min-max shortest $s$-t-paths in $G\left(u_{j_{0}}\right)$, i.e., it is

$$
\max _{r \in S} \tau^{r}\left(P_{j_{0}}\right)=\max _{r \in S} \tau^{r}(P)
$$

By definition of $G\left(u_{j_{0}}\right)$, it is $u\left(P_{j_{0}}\right) \geq u(P)$ and, thus,

$$
\max _{r \in S} \tau^{r}\left(P_{j_{0}}\right)+\left\lceil\frac{U}{u\left(P_{j_{0}}\right)}\right\rceil \leq \max _{r \in S} \tau^{r}(P)+\left\lceil\frac{U}{u(P)}\right\rceil .
$$

Assumption (2) implies

$$
\max _{r \in S} \sigma^{r}\left(P_{k}\right) \leq \max _{r \in S} \sigma^{r}(P)
$$

which shows that $P_{k}$ is a min-max quickest $s$-t-path in $G$.

Corollary 3. MIN-MAX $\mathcal{Q}^{\tau}$ can be solved in $\mathcal{O}\left(m n^{2} B^{p-1}\right)$.

Proof. Lemma 5 is constructive for the min-max quickest $s$ - $t$-path. At most $m$ min-max shortest path problems have to be solved, each of which requires $\mathcal{O}\left(n^{2} B^{p-1}\right)$ time.

\subsection{Fully Polynomial-Time Approximation Scheme for MIN-MAX $\mathcal{Q}^{\tau}$}

For the min-max shortest path problem, the following approximation result was published in Aissi et al. [2].

Lemma 6. [Aissi et al. [2]] The min-max shortest $s$-t-path problem for $p$ scenarios admits an fptas running in time $\mathcal{O}\left(\frac{n^{p+1}}{\varepsilon^{p-1}}\right)$.

A similar approximation result can be proved for MIN-MAX $\mathcal{Q}^{\tau}$.

Lemma 7. Let $\varepsilon>0$. Let $P_{j}$ be a $(1+\varepsilon)$-approximation of the min-max shortest $s$-t-path in $G\left(u_{j}\right)$ for all $j=1, \ldots, l$. Let

$$
P_{k} \in \underset{j=1, \ldots, l}{\operatorname{argmin}} \max _{r \in S}\left(\tau^{r}\left(P_{j}\right)+\left\lceil\frac{U}{u\left(P_{j}\right)}\right\rceil\right) .
$$

Then, $P_{k}$ is a $(1+\varepsilon)$-approximation of the min-max quickest s-t-path in $G$.

Proof. Let $Q$ be a solution of MIN-Max $\mathcal{Q}^{\tau}$ in $G$. There is some $j_{0} \in\{1, \ldots, l\}$ with $u_{j_{0}}=u(Q)$. Due to Lemma $4, Q$ is a min-max shortest path in $G\left(u_{j_{0}}\right)$. Thus,

$$
\max _{r \in S} \tau^{r}\left(P_{j_{0}}\right) \leq(1+\varepsilon) \max _{r \in S} \tau^{r}(Q) .
$$


With $u\left(P_{j_{0}}\right) \geq u(Q)$, it follows

$$
\begin{aligned}
\max _{r \in S} \tau^{r}\left(P_{j_{0}}\right)+\left\lceil\frac{U}{u\left(P_{j_{0}}\right)}\right\rceil & \leq(1+\varepsilon) \max _{r \in S} \tau^{r}(Q)+\left\lceil\frac{U}{u(Q)}\right\rceil \\
& \leq(1+\varepsilon)\left(\max _{r \in S} \tau^{r}(Q)+\left\lceil\frac{U}{u(Q)}\right\rceil\right) .
\end{aligned}
$$

Using the definition, one concludes that $P_{k}$ is a $(1+\varepsilon)$-approximation of the min-max quickest $s$-t-path.

Corollary 4. MIN-MAX $\mathcal{Q}^{\tau}$ admits an fptas running in $\mathcal{O}\left(\frac{m n^{p+1}}{\varepsilon^{p-1}}\right)$

Proof. Using that Lemma 7 is constructive for a $(1+\varepsilon)$-approximative solution of MIN-MAX $\mathcal{Q}^{\tau}$, the complexity follows directly from Lemma 6 .

\section{Scenario-Dependent Travel Times and Capacities}

Consider the problem with travel times and capacities both being scenariodependent. Obviously, the corresponding min-max problem subsumes the problems MIN-MAX $\mathcal{Q}^{\tau}$ and, thus, it is NP-hard due to Corollary 2.

To solve MIN-MAX $\mathcal{Q}_{u}^{\tau}$, a related multiple objective optimization problem is introduced. It will be shown that a $(1+\varepsilon)$-approximation of the efficient set (i.e. the optimal set) of this multiple objective optimization problem yields a $(1+\varepsilon)$-approximation of MIN-MAX $\mathcal{Q}_{u}^{\tau}$. Before proving this result, some basic concepts from multiple objective optimization are stated next. The book of Ehrgott [6] provides a more detailed introduction on this topic.

Let $\min _{x \in X}\left(f^{1}(x), \ldots, f^{p}(x)\right)$ be a multiple objective optimization problem with feasible solution set $X$ and objective functions $f^{1}, \ldots, f^{p}$ to be minimized simultaneously. Note that there is no canonical ordering defined on $\mathbb{R}^{p}$. A "better-than"-relation implying an optimality concept has therefore to be defined. Given two solutions $x, y \in X, x$ dominates $y$ if $f^{r}(x) \leq f^{r}(y)$ for all $r \in\{1, \ldots, p\}$ with at least one strict inequality. A solution $x \in X$ is efficient if $x$ is not dominated by some $y \in X$. The set of efficient solutions is denoted by $X_{E}$. A solution $x$ is weakly efficient if there is no $y \in X$ such that $f^{r}(y) \leq f^{r}(x)$ for all $r \in\{1, \ldots, p\}$. The set of weakly efficient solutions is called the weakly efficient set. For $\varepsilon>0$, a $(1+\varepsilon)$-approximation of the efficient set $X_{\varepsilon}$ is a subset of $X$ such that for all $x^{\prime} \in X_{E}$ there is some $x \in X_{\varepsilon}$ with $f^{r}(x) \leq(1+\varepsilon) f^{r}\left(x^{\prime}\right)$ for all $r \in\{1, \ldots, p\}$.

Consider the following multiple objective optimization problem with $2 p=2|S|$ objective functions:

$(M O P)$

$$
\min _{P \in \mathcal{P}}\left(\tau^{1}(P),\left\lceil\frac{U}{u^{1}(P)}\right\rceil, \ldots, \tau^{p}(P),\left\lceil\frac{U}{u^{p}(P)}\right\rceil\right) .
$$


In this auxiliary problem, it is $X=\mathcal{P}$. Therefore, the notation $\mathcal{P}_{E}$ and $\mathcal{P}_{\varepsilon}$ is used to refer to the set of efficient paths of MOP and to a $(1+\varepsilon)$ approximation of $\mathcal{P}_{E}$, respectively.

Obviously, every solution of MIN-MAX $\mathcal{Q}_{u}^{\tau}$ is weakly efficient for MOP. For the converse case, the following is true.

Lemma 8. There is at least one optimal solution of MIN-MAX $\mathcal{Q}_{u}^{\tau}$ which is efficient for MOP.

Proof. Assume $Q$ is an optimal solution of MIN-MAX $\mathcal{Q}_{u}^{\tau}$ not being efficient for MOP. Then, there is an efficient path $P \in \mathcal{P}_{E}$ which dominates $Q$, i.e., $\tau^{r}(P) \leq \tau^{r}(Q)$ and $\left\lceil\frac{U}{u^{r}(P)}\right\rceil \leq\left\lceil\frac{U}{u^{r}(Q)}\right\rceil$ for all $r \in S$ and there is a $j \in S$ with $\tau^{j}(P)<\tau^{j}(Q)$ or $\left\lceil\frac{U}{u^{j}(P)}\right\rceil<\left\lceil\frac{U}{u^{j}(Q)}\right\rceil$. Therefore, for all $r \in S$, it holds

$$
\tau^{r}(P)+\left\lceil\frac{U}{u^{r}(P)}\right\rceil \leq \tau^{r}(Q)+\left\lceil\frac{U}{u^{r}(Q)}\right\rceil
$$

and, as a consequence, it is

$$
\max _{r \in S}\left(\tau^{r}(P)+\left\lceil\frac{U}{u^{r}(P)}\right\rceil\right) \leq \max _{r \in S}\left(\tau^{r}(Q)+\left\lceil\frac{U}{u^{r}(Q)}\right\rceil\right) .
$$

Thus, $P$ solves Min-max $\mathcal{Q}_{u}^{\tau}$.

Lemma 9. For $\varepsilon>0$, let $\mathcal{P}_{\varepsilon}$ be a $(1+\varepsilon)$-approximation of the efficient set for $M O P$. If $\mathcal{P}_{\varepsilon}$ can be constructed in time polynomial in the input size and $1 / \varepsilon$, then MIN-MAX $\mathcal{Q}_{u}^{\tau}$ admits an fptas.

Proof. Assume $Q$ solves MIN-MAx $\mathcal{Q}_{u}^{\tau}$ and is efficient for MOP (Lemma 8).

Then, there is a $P \in \mathcal{P}_{\varepsilon}$ with

$$
\begin{aligned}
& \tau^{r}(P) \leq(1+\varepsilon) \tau^{r}(Q) \quad \text { for all } r \in S \text { and } \\
&\left\lceil\frac{U}{u^{r}(P)}\right\rceil \leq(1+\varepsilon)\left\lceil\frac{U}{u^{r}(Q)}\right\rceil \quad \text { for all } r \in S .
\end{aligned}
$$

Then,

$$
\begin{aligned}
\tau^{r}(P)+\left\lceil\frac{U}{u^{r}(P)}\right\rceil & \leq(1+\varepsilon)\left(\tau^{r}(Q)+\left\lceil\frac{U}{u^{r}(Q)}\right\rceil\right) \quad \text { for all } r \in S . \\
\Rightarrow \max _{r \in S}\left(\tau^{r}(P)+\left\lceil\frac{U}{u^{r}(P)}\right\rceil\right) & \leq \max _{r \in S}(1+\varepsilon)\left(\tau^{r}(Q)+\left\lceil\frac{U}{u^{r}(Q)}\right\rceil\right) \\
& =(1+\varepsilon) \max _{r \in S}\left(\tau^{r}(Q)+\left\lceil\frac{U}{u^{r}(Q)}\right\rceil\right) .
\end{aligned}
$$


Thus, $P$ is a $(1+\varepsilon)$-approximation of $Q$. Since $\mathcal{P}_{\varepsilon}$ can be constructed in time polynomial in the input size and in $1 / \varepsilon$, the size of $\mathcal{P}_{\varepsilon}$ must bounded by a polynomial of the same size and, thus, $P$ can be found in time polynomial in the input size and $1 / \varepsilon$.

It remains to be shown that $\mathcal{P}_{\varepsilon}$ for MOP has a computation time complexity bounded by a polynomial in the input size and $1 / \varepsilon$.

For each scenario $r \in S$, let $u_{1}^{r}<u_{2}^{r}<\ldots<u_{l_{r}}^{r}$ be the distinct capacities in this scenario with $l_{r} \leq m$. For $k_{r} \in\left\{1, \ldots, l_{r}\right\}, r \in S$, consider the network $G\left(k_{1}, \ldots, k_{p}\right)$ with $\operatorname{arcs} A\left(k_{1}, \ldots, k_{p}\right):=\left\{(i, j) \in A: u_{i j}^{r} \geq u_{r}^{k_{r}} ; \forall r \in\right.$ $S\}$. Denote by $\mathcal{P}_{k_{1}, \ldots, k_{p}}$ the set of all $s$-t-paths in $G\left(k_{1}, \ldots, k_{p}\right)$. Consider the multiple objective shortest path problem $\min _{P \in \mathcal{P}_{k_{1}, \ldots, k_{p}}}\left(\tau^{1}(P), \ldots, \tau^{p}(P)\right)$ and compute a $(1+\varepsilon)$-approximation of the efficient set $\mathcal{P}_{\varepsilon}^{k_{1}, \ldots, k_{p}}$ for this problem.

Define the set

$$
\mathcal{P}_{\varepsilon}:=\bigcup_{r \in S} \bigcup_{k_{r} \in\left\{1, \ldots, l_{r}\right\}} \mathcal{P}_{\varepsilon}^{k_{1}, \ldots, k_{p}}
$$

Lemma 10. $\mathcal{P}_{\varepsilon}$ can be constructed in time polynomial in the input size and $1 / \varepsilon$.

Proof. Each of the approximations $\mathcal{P}_{\varepsilon}^{k_{1}, \ldots, k_{p}}$ can be constructed in time polynomial in the input size and $1 / \varepsilon$ due to the work of Papadimitriou and Yannakakis [15]. The number of combinations of $k_{1}, \ldots, k_{p}$ is

$$
\prod_{r \in S} l_{r} \leq \prod_{r \in S} m=m^{p}
$$

and, thus, the time complexity for the construction of $\mathcal{P}_{\varepsilon}$ is bounded by a polynomial in the input size and $1 / \varepsilon$.

Lemma 11. Given $\varepsilon>0, \mathcal{P}_{\varepsilon}$ is a $(1+\varepsilon)$-approximation of the efficient set for $M O P$.

Proof. Let $\bar{P}$ be an efficient solution of MOP. Then there are $u_{1}^{k_{1}}, \ldots, u_{p}^{k_{p}}$ with $\left\lceil\frac{U}{u_{r}^{k_{r}}}\right\rceil=\left\lceil\frac{U}{u^{r}(\bar{P})}\right\rceil$ for all $r \in S$. Consider the network $\widetilde{G}:=G\left(k_{1}, \ldots, k_{p}\right)$. Assume that $\bar{P}$ is not efficient for $\min _{P \in \mathcal{P}_{k_{1}, \ldots, k_{p}}}\left(\tau^{1}(P), \ldots, \tau^{p}(P)\right)$. Then there exists a $Q \in \mathcal{P}_{k_{1}, \ldots, k_{p}}$ with $\tau^{r}(Q) \leq \tau^{r}(\bar{P})$ for all $r \in S$ with strict inequality for at least one $r \in S$. It is $u^{r}(Q) \geq u^{r}(\bar{P})$ for all $r \in S$ and thus $\left\lceil\frac{U}{u^{r}(Q)}\right] \leq\left[\frac{U}{u^{r}(\bar{P})}\right\rceil$ for all $r \in S$. This implies that $Q$ dominates $\bar{P}$ for MOP which is a contradiction. 
By definition, there exists $Q \in \mathcal{P}_{\varepsilon} \cap \mathcal{P}_{\varepsilon}^{k_{1}, \ldots, k_{p}}$ with $\tau^{r}(Q) \leq(1+\varepsilon) \tau^{r}(\bar{P})$ for all $r \in S$. Since $Q$ is a path in $\widetilde{G}$, it is $u^{r}(Q) \geq u^{r}(\bar{P})$ for all $r \in S$ and, thus,

$$
\left\lceil\frac{U}{u^{r}(Q)}\right\rceil \leq\left\lceil\frac{U}{u^{r}(\bar{P})}\right\rceil \leq(1+\varepsilon)\left\lceil\frac{U}{u^{r}(\bar{P})}\right\rceil \text { for all } r \in S .
$$

Hence, $Q$ approximates $\bar{P}$ within a factor of $(1+\varepsilon)$ for MOP.

Let $\tau^{\max }$ be the ratio of the maximum to the minimum travel time in all scenarios.

Corollary 5. MIN-MAX $\mathcal{Q}_{u}^{\tau}$ admits an fptas running in time

$\mathcal{O}\left(m^{p+1} n\left(\frac{n \log n \tau^{\max }}{\varepsilon}\right)^{p-1}\right)$.

Proof. Tsaggouris and Zaroliagis [18] show that $(1+\varepsilon)$-approximations of the efficient sets for multiple objective shortest path problems can be constructed with computation time $\mathcal{O}\left(n m\left(n \log \left(n \tau^{\max }\right) / \varepsilon\right)^{p-1}\right)$.

The size of these $(1+\varepsilon)$-approximations of the efficient sets is bounded by $\mathcal{O}\left(n m\left(n \log \left(n \tau^{\max }\right) / \varepsilon\right)^{p-1}\right)$. Thus, the complexity to compute a $(1+\varepsilon)$ approximation of MIN-MAX $\mathcal{Q}_{u}^{\tau}$ follows from Lemma 10.

Note that this result also implies an fptas for MIN-MAX $\mathcal{Q}^{\tau}$, yet with a running time worse than the one of the algorithm presented in Section 4.

\section{Conclusion and Future Research}

This article introduces min-max versions of the well-known quickest path problem. Several different scenario-dependent parameter configurations are examined. For uncertain capacities, an algorithm is proposed with computational complexity similar to that of the quickest path problem. In the case of scenario-dependent travel times, the min-max quickest path problem is shown to be NP-hard and a pseudopolynomial algorithm as well as a fully polynomial-time approximation scheme is derived. A fully polynomialtime approximation scheme is also proposed for the min-max quickest path problem with scenario-dependent capacities and travel times.

Future research might address a related problem, the so-called Min-Max Regret Quickest Path Problem. Let $z^{r}:=\min _{P \in \mathcal{P}} \sigma^{r}(P)$ denote the optimal objective function value for scenario $r \in S$. The Min-Max Regret Quickest Path Problem is $\min _{P \in \mathcal{P}} \max _{r \in S}\left(\sigma^{r}-z^{r}\right)$. Here, a path $P \in \mathcal{P}$ minimizing the maximum relative error ("regret") among all scenarios is demanded. 
Some of the techniques used in this article directly carry over to the corresponding min-max regret versions. The min-max regret version of $\mathcal{Q}_{u}$ can be solved exactly in polynomial time by a single quickest path computation in a modified network using a similar technique applied in Section 3 for the corresponding min-max problem. Since the min-max regret shortest path problem is NP-hard ( $\mathrm{Yu}$ and Yang [20]), the min-max regret version of $\mathcal{Q}^{\tau}$ is also an NP-hard problem. It can be solved in pseudopolynomial time combining the pseudopolynomial algorithm for the min-max regret shortest path problem of Aissi et al. [2] with arguments similar to those of Section 4.1 .

The existence of approximation schemes for the min-max regret problem with scenario-dependent travel times as well as with scenario-dependent travel times and capacities is still open since the ideas and techniques used in this article cannot be directly applied due to the existence of the additive term $z^{r}$ in this case.

Further research should also concentrate on robust versions of other (dynamic) network flow problems, including maximum (dynamic) flows, quickest flows, and minimum cost flows.

\section{References}

[1] H. Aissi, C. Bazgan, and D. Vanderpooten. Min-max and min-max regret versions of combinatorial optimization problems: A survey. European Journal of Operational Research, 197(2):427-438, 2009.

[2] H. Aissi, C. Bazgan, and D. Vanderpooten. General approximation schemes for min-max (regret) versions of some (pseudo-) polynomial problems. Discrete Optimization, 7(3):136-148, 2010.

[3] Y.L. Chen. Finding the $\mathrm{k}$ quickest simple paths in a network. Information Processing Letters, 50(2):89-92, 1994.

[4] Y.L. Chen and Y.H. Chin. The quickest path problem. Computers 85 Operations Research, 17(2):153-161, 1990.

[5] J.C.N. Clímaco, M.M.B. Pascoal, J.M.F. Craveirinha, and M.E.V. Captivo. Internet packet routing: Application of a K-quickest path algorithm. European Journal of Operational Research, 181(3):1045-1054, 2007.

[6] M. Ehrgott. Multicriteria optimization. Springer, Berlin, 2005.

[7] H.W. Hamacher and S.A. Tjandra. Mathematical modelling of evacuation problems-a state of the art. In Pedestrian and Evacuation Dynamics, pages 227-266. Springer, Berlin, 2002. 
[8] Y.-C. Hung and G.-H. Chen. On the quickest path problem. Lecture Notes in Computer Science, 497:43-46, 1991.

[9] P. Kouvelis and G. Yu. Robust discrete optimization and its applications. Kluwer Academic Publishers, Dordrecht, 1997.

[10] D.T. Lee and E. Papadopoulou. The all-pairs quickest path problem. Information Processing Letters, 45(5):261-267, 1993.

[11] Y.K. Lin. Extend the quickest path problem to the system reliability evaluation for a stochastic-flow network. Computers $\&$ Operations Research, 30(4):567-575, 2003.

[12] E.Q.V. Martins and J.L.E. dos Santos. An algorithm for the quickest path problem. Operations Research Letters, 20(4):195-198, 1997.

[13] M.H. Moore. On the fastest route for convoy-type traffic in flowrateconstrained networks. Transportation Science, 10(2):113-124, 1976.

[14] C.H. Papadimitriou and K. Steiglitz. Combinatorial optimization: algorithms and complexity. Prentice-Hall Inc., Englewood Cliffs, NJ, 1982.

[15] C.H. Papadimitriou and M. Yannakakis. On the approximability of trade-offs and optimal access of web sources (Extended Abstract). In Proceedings 41st Annual Symposium on Foundations of Computer Science, pages 86-92, 2000.

[16] M.M.B. Pascoal, M.E.V. Captivo, and J.C.N. Clímaco. A comprehensive survey on the quickest path problem. Annals of Operations Research, 147(1):5-21, 2006.

[17] J.B. Rosen, S.Z. Sun, and G.L. Xue. Algorithms for the quickest path problem and the enumeration of quickest paths. Computers $\&$ Operations Research, 18(6):579-584, 1991.

[18] G. Tsaggouris and C. Zaroliagis. Multiobjective optimization: Improved FPTAS for shortest paths and non-linear objectives with applications. Theory of Computing Systems, 45(1):162-186, 2009.

[19] G. Xue. End-to-end data paths: quickest or most reliable? IEEE Communications Letters, 2(6):156-158, 1998.

[20] G. Yu and J. Yang. On the robust shortest path problem. Computers \&6 Operations Research, 25(6):457-468, 1998. 